\title{
revista.traidumà̀tica
}

147

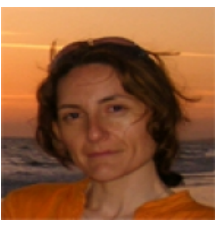

\section{El uso de blogs y wikis en la formación del traductor}

María Dolores García Santiago

Departamento de Información y Comunicación

Universidad de Granada

\section{RESUMEN}

En el artículo se describe el proyecto de innovación docente universitario consistente en el uso y aplicación de una wiki para la adquisición de competencias dentro de la formación y actualización del traductor. Se utilizó un cuestionario para analizar el aprendizaje adquirido y los hábitos de uso de servicios de la Web 2.0. El valor innovador de esta iniciativa mejora la formación y sirve como modelo de enseñanza.

Palabras clave: aprendizaje colaborativo, Web 2.0, wikis, traducción e interpretación, innovación docente, e-learning, blogs, subcompetencia informacional

\section{ABSTRACT}

This paper describes an innovative educational project at University. It lies in using and applying a wiki. The aim is to acquire competencies of translating studies. A questionnaire analized the learning level reached and the use habits of Web 2.0 tools. The innovation value of this initiative improves training and helps as teaching model.

Keywords: collaborative learning, Web 2.0, wikis, translation and interpreting studies, innovative educational project, e-learning, blogs, information competence

\section{RESUM}

En l'article es descriu el projecte d'innovació docent universitari consistent en l'ús i aplicació d'una wiki per a l'adquisició de competències dins de la formació i actualització del traductor. Un qüestionari va servir per analitzar l'aprenentatge adquirit i els hàbits d'ús de serveis de la web 2.0. El valor innovador d'aquesta iniciativa millora la formació i serveix com a model d'ensenyament.

Paraules clau: aprenentatge col·laboratiu, Web 2.0, wikis, traducció i interpretació, innovació docent, e-learning, blogs, subcompetència informacional

\section{Introducción}

La traducción y la interpretación son actividades con un alto número de profesionales autónomos (en inglés freelance) que necesitan estar en constante renovación y actualización. Este aprendizaje comienza en los estudios de educación superior de esta especialidad. Se enseñan los aspectos lingüísticos y metodológicos para transmitir mensajes de una lengua a otra sin tergiversarlos, y sin alterar la intención del autor/a. También se contemplan las competencias informacionales que les capaciten para identificar, localizar, buscar y recuperar recursos informativos; además de usar herramientas ofimáticas y otras tecnologías que les permitan elaborar traducciones de calidad.

Desde esta perspectiva tecnológica, los actuales estudiantes universitarios son nativos digitales. Han nacido y crecido a la par que Internet; con una visión a tiempo real y en línea a través de estas tecnologías (Zickuhr, 2010), con procesos de razonamiento y socialización diferentes. Sin embargo, eso no los convierte en expertos de la Web 2.0, ese grupo de tecnologías en línea que facilitan la conexión social, y donde cada persona es capaz de crear y/o editar información a través de Internet. Siguiendo la taxonomía de Bloom (Churches, 2009), todo/a estudiante puede contar con aptitudes para: memorizar, entender, aplicar, 
analizar, evaluar y crear. Hoy día, utilizando herramientas de la Web 2.0, se pueden encontrar en cualquiera de estos niveles (Penney, 2012).

La Universidad, dentro del Espacio Europeo de Educación Superior, debe subsanar estas carencias en el contexto docente. Con el fomento del interés y la aplicación de estas tecnologías en la profesión, especialmente en la traducción y la interpretación, se formarán profesionales competentes (Alcina Caudet, 2002; Pérez González y Rico Pérez, 2001). El teletrabajo colaborativo obliga al traductor integrado en un equipo de traducción a manejar redes sociales y otros servicios de la Web 2.0 para una mejor comunicación y un mayor rendimiento en el producto final (Olvera Lobo et al, 2007 y Martín y Soler, 2005).

\section{El proyecto TradWiki}

Es fruto de la experiencia llevada a cabo por los estudiantes de la asignatura "Documentación Científica y Técnica" dentro de los estudios de Traducción e Interpretación de la Universidad de Granada. Se apoya en una plataforma de aprendizaje en línea, para una formación tanto presencial como virtual y en la que se integran recursos de la Web 2.0 como potenciales herramientas para la optimización del trabajo del traductor y del equipo de traducción (Sturm, et al, 2009; Polo y Ferrer Mora, 2010).

Esta asignatura optativa complementa a la materia básica y obligatoria "Documentación aplicada a la traducción". Ambas se centran en la alfabetización informacional siendo más especializada la materia "Documentación Científica y Técnica" y profundizando en herramientas de la Web 2.0 especialmente entre aquellos que, de manera natural, no tienen un especial interés por la informática.

Se escogieron los servicios Web 2.0 implantados de forma más generalizada. El blog o bitácora como recurso web de información personal, con uno o varios autores, de actualización periódica; y la wiki. Aunque existen ya experiencias con wikis y/o blogs (Zayas, 2006; Duffy y Axel, 2006; Bold, 2006), este experiencia pretendió dar un mayor énfasis a la aplicación profesional.

La wiki es una aplicación informática en línea ubicado en un servidor web y que permite crear y editar contenido de página web a través de cualquier tipo de navegador (Leuf y Cunningham, 2002). Es decir, una base de datos cooperativa que cuenta con una sintaxis muy sencilla que permite crear en cualquier momento nuevas páginas web con hiperenlaces que las relacionan. El hecho de que sea en abierto permite que cualquiera sin conocimientos técnicos pueda modifique cualquier contenido.

Esta wiki para traducción, TradWiki, fue mantenida y desarrollada por los estudiantes con el objetivo de adquirir el conocimiento teórico y aplicado sobre esta herramienta (Lavin y Claro, 2005) dentro de su formación como traductores. Es decir, utilizarla para:

- Conocer su estructura y funcionamiento.

- Realizar tareas de traducción: realizando búsquedas de documentación técnica para alimentar la wiki y generando una fuente terminológica colectiva procedente de fuentes fiables.

- Facilitar la edición, presentación y revisión de traducciones como si de un equipo de traducción se tratara.

- Compartir conocimientos con el resto de compañeros con análisis y valoraciones de blogs sobre traducción.

- Difundir contenidos de la Web 2.0 en otros formatos como vídeos de plataformas como Youtube, Vimeo...

- Fomentar la comunicación entre todos/as los miembros del grupo.

Desde una perspectiva constructivista, se organizan tareas individuales para que cada estudiante, como miembro de un equipo, lleve a cabo el proceso de traducción aplicando sus conocimientos, habilidades y competencias. Así se consigue mejorar un producto final en constante actualización como es la propia TradWiki, que servirá de base para ampliar el conocimiento de futuros lectores. 


\section{Metodología}

\subsection{Diseño}

TradWiki está integrada en la plataforma de enseñanza virtual Moodle, con acceso restringido a la consulta y edición sólo para los miembros de la asignatura (véase fig. 1).

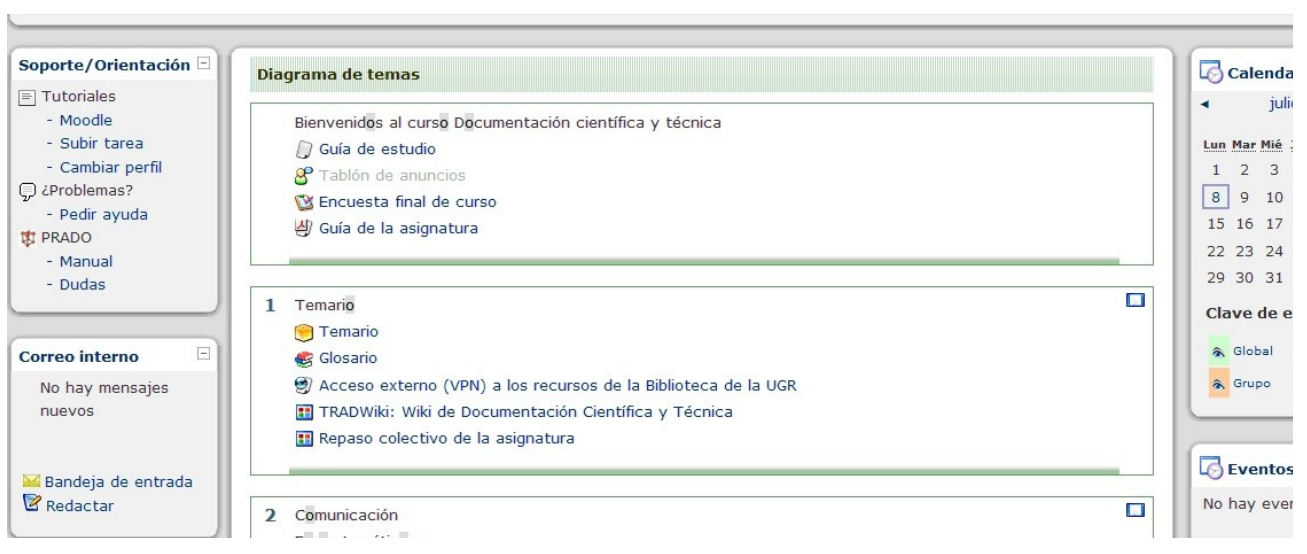

Figura 1: Página principal de la asignatura en Moodle

Las tareas a realizar por el estudiante a través de TradWiki corresponden a las diferentes fases del proceso de traducción. Estas se dividieron en función de la dificultad y conocimientos mínimos para realizarlas.

- Tareas obligatorias: encaminadas a un conocimiento básico pero suficiente del funcionamiento de una wiki, su edición y del trabajo colaborativo.

- Tareas optativas: pretendían cubrir la curiosidad de las personas con mayor iniciativa y participación.

Estas tareas se materializan en un listado que formaba parte de la estructura de TradWiki. En un principio, nuestra wiki parte de un número mínimo de elementos consecuencia de las tareas obligatorias solicitadas, que va creciendo tanto como el interés y ánimo de sus participantes. Esto se debe a su gran sencillez técnica, donde sólo es necesario crear un enlace sobre un término concreto para generar una página editable en la que se incluya contenido (Sigala, 2007; Franklin y Van Harmelen, 2007).

La facilidad de conservar el historial de los cambios realizados, permite al administrador de la wiki recuperar fácilmente cualquier estado anterior de la página, identificar la autoría y controlar el grado de participación. Finalmente se obtuvo el documento colaborativo deseado en el que todo el grupo participó compartiendo conocimientos, traducciones y revisándolas, buscando nueva información y valorando otros servicios de comunicación como son los blogs sobre traducción e interpretación.

\subsection{Estructura}

Los apartados que forman el índice reflejan, principalmente, aspectos de las etapas pretraductora con fuentes de información y recursos terminológicos, y traductora (Olvera Lobo et al, 2004). En cada uno de ellos se planteaban las actividades que permitían cubrir las potenciales necesidades de un traductor. Cualquier estudiante podía realizar estas actividades que podían ser revisadas y editadas por el resto de los/as participantes. Las secciones que se incluían eran las siguientes:

- Vocabulario específico: Se ofertó un listado terminológico inicial que impelía a la búsqueda terminológica. El trabajo de cada estudiante localizando la definición de un 
solo término, genera un pequeño y particular glosario (fig.2). A este apartado se le pueden agregar los términos que cada persona, ya voluntariamente quisiera añadir.

- Diccionarios en línea: Se partía de un mínimo de recursos terminológicos, mayoritariamente diccionarios. Además, se invitó a la inclusión de más fuentes terminológicas accesibles a través de la Web. También se reunió un aceptable número de diccionarios en las lenguas mayoritarias.

- Blogs sobre Traducción e Interpretación: Se suministró una considerable cantidad de blogs. Cada persona debía acceder, como mínimo, a uno de ellos e incluir un comentario crítico sobre el contenido del mismo y justificar el potencial interés para un traductor. Se lograba así un doble fin, por un lado el conocimiento de nuevas fuentes de información y servicios Web 2.0; y por otra, desarrollar la capacidad crítica valorando elementos del blog en cuestión.

- Traducción colectiva de una patente: En esta sección, se trataba de mostrar el trabajo de un traductor especializado. Se llevó a cabo el proceso de traducción completo, incluida la fase de revisión y la comparación de textos paralelos al ser una traducción técnica a varios idiomas, en función de la especialidad de los alumnos. Primero se traducía un documento largo de manera cooperativa. Para esto se subdividió el texto por fragmentos para evitar sobrecargas de trabajo. Se tradujeron simultáneamente, ya que cada persona trabajaba en paralelo lo que permite una mayor celeridad generar el documento final. Por último, otro/a compañero/a revisaba el documento para completar el proceso.

- Otros apartados: Denominados Vídeos, Noticias y Humor relacionados con la traducción y la interpretación se añadieron para fomentar el interés y la participación. Con ello se retoma la intención primigenia de una wiki, la cooperación de manera informal.

Para una mejor coordinación entre compañeros, las comunicaciones se realizaron a través de un foro específico integrado en la plataforma Moodle.

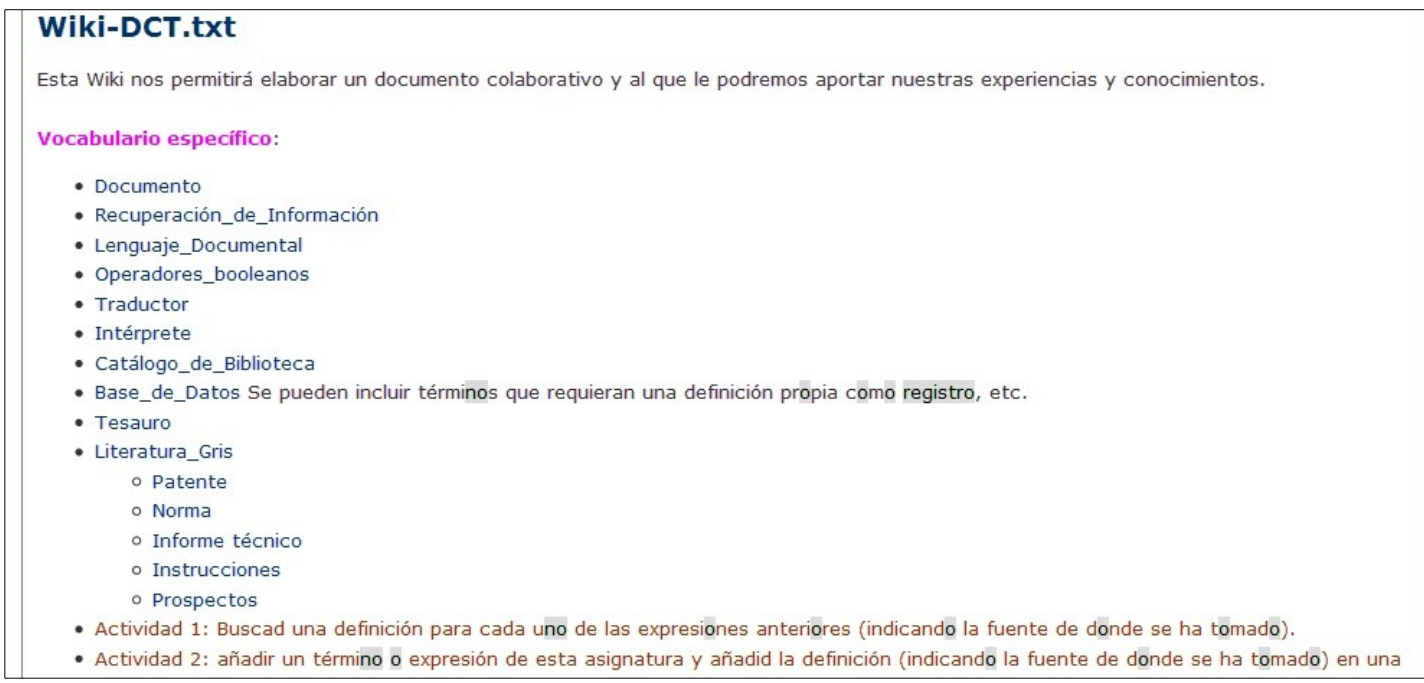

Figura 2: Apartado y actividades dentro de la wiki

\subsection{Análisis y evaluación}

Se valoró en qué medida se han alcanzado unos objetivos de aprendizaje previamente establecidos. Para ello se confeccionaron dos cuestionarios que se integraron en la plataforma Moodle, y que recogían las impresiones personales de aprendizaje, las dificultades y también los prejuicios que les impedían adquirir nuevas destrezas con herramientas de la Web 2.0. 
El cuestionario inicial, se centró en los hábitos tecnológicos, especialmente referidos a la Web 2.0. Mientras que la encuesta final recogía observaciones y sugerencias personales y aspectos relativos al aprendizaje con la wiki. Con la comparación de ambas encuestas se identificaron nuevos o cambios de hábitos con respecto a wikis y blogs,.

Paralelamente, el histórico de ediciones permitió el seguimiento y análisis de las acciones realizadas por cada estudiante con la wiki. Finalmente, se evaluó el aprendizaje a nivel global de la asignatura y se identificaron y valoraron las actividades realizadas en la wiki.

\section{Resultado}

Los 12 alumnos que cursaron la asignatura participaron activamente en las actividades del proyecto TradWiki.

El primer cuestionario constaba de 19 preguntas (anexo 1) y tenía como objetivo identificar los hábitos de uso y los conocimientos iniciales sobre la Web 2.0 antes de comenzar a trabajar con TradWiki.

En relación con los hábitos generales, la totalidad del alumnado utiliza asiduamente Internet y algunos recursos de la Web 2.0. Se confirma su conocimiento sobre las redes sociales como Twitter, Facebook o Linkedin aunque existe una heterogeneidad a la hora de utilizar otras herramientas de la Web 2.0. De estos, un 50\% conocía de la existencia de otro recurso diferente de la Web 2.0 como Dropbox, Flickr, Google+, Linkedln, Seriesly, Spotify, Studivz, Swad, Tumblr, Vimeo y Wordpress. Pero sólo un 17\% habían utilizado: Seriesly, Tumblr, Vimeo y Wordpress con dispar frecuencia. Estos datos confirman el acierto de crear TradWiki para reforzar este tipo de formación.

Tanto en el ámbito especializado de la traducción y la interpretación como a nivel general, sólo un $50 \%$ de los estudiantes consultan blogs (véase anexo 2) y a pesar de contar con la información sobre ellos. Destacar que únicamente una persona participaba activamente en ellos.

En el caso de las wikis, aparte de Wikipedia, conocida por el $100 \%$ de los participantes, sólo se mencionaron Wikilengua y Wikitravel en menor medida. No obstante, conocían mayoritariamente las funciones generales de una wiki con su capacidad de creación y edición de manera instantánea.

El desarrollo de TradWiki propiamente dicho ha sido muy positivo. Todas las creaciones y ediciones son recogidas por la wiki, indicando cuándo y qué información ha sido modificada. La figura 3 muestra los alumnos y aportaciones por sección, que varían en relación directa con los conocimientos técnicos.

Figura 3: Resumen de las participaciones de estudiantes en TradWiki 


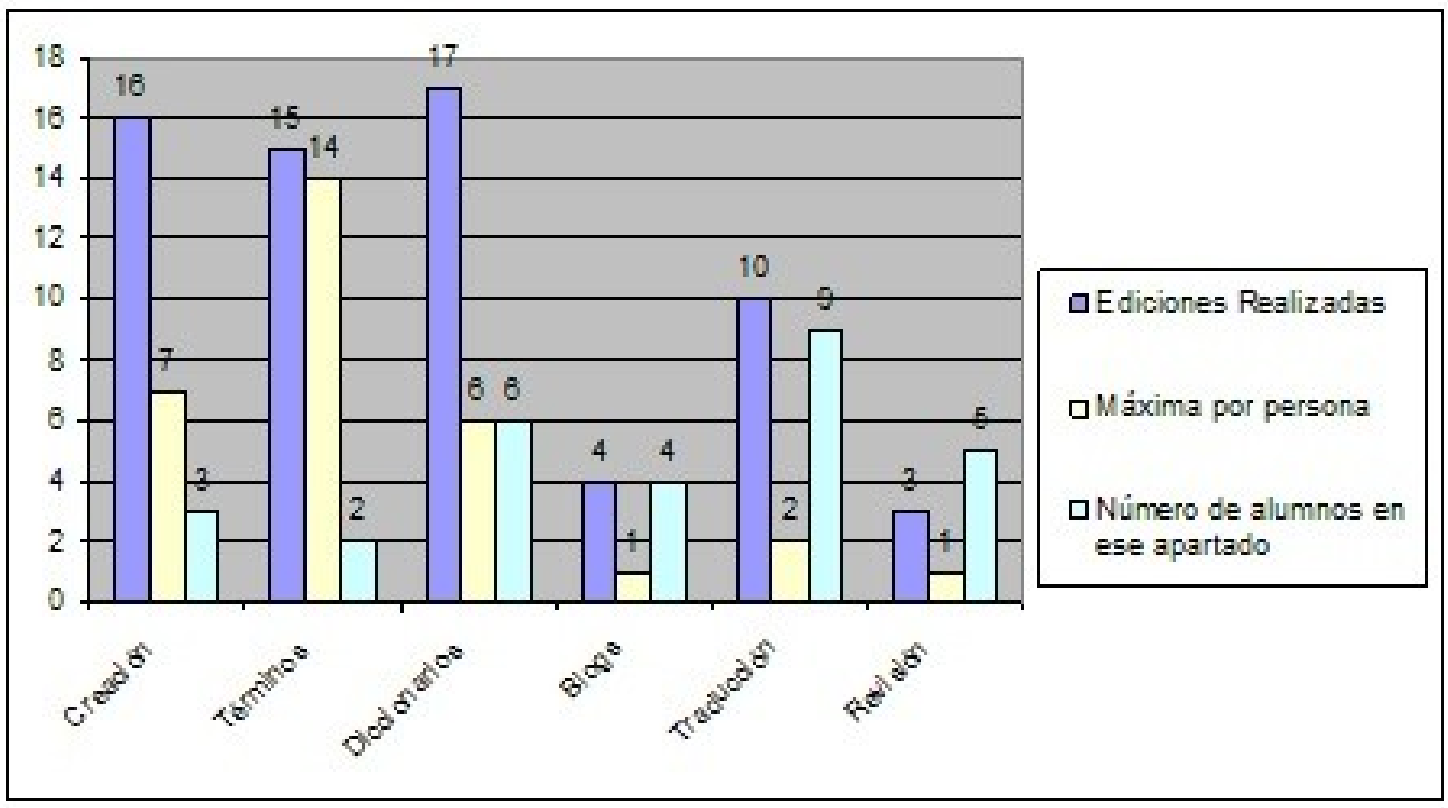

Se ha conseguido una participación muy aceptable con una media de 5 ediciones por persona. Tan sólo dos personas $(16,6 \%)$ han realizado una única edición y otra $(8,3 \%)$ ha llegado a una máxima de 24 ediciones. Estos datos reflejan claramente el grado de implicación e interés por parte de los/as alumnos/as (75\%) en el ejercicio al superar los mínimos planteados en la actividad, sobre todo en la memoria colectiva de fuentes de información terminológica.

Una encuesta final recogió las impresiones y opiniones de los alumnos sobre el uso y utilidad de wikis y blogs (anexo 3). El 100\% de los participantes consideró útil o muy útil el uso de una wiki en su profesión como traductores debido a que:

- Facilita trabajo colaborativo y en equipo

- Es una excelente herramienta de revisión

- Mejora el aprendizaje con el trabajo en equipo en sí mismo y corrigiendo errores ajenos

- Permite realizar proyectos de traducción con flexibilidad geográfica y tempora

- Ahorra tiempo de trabajo.

- Aporta ayuda y nuevos conocimientos del y al resto de los miembros como la forma de redactar.

- Permite compartir el documento, visualizando la última versión a todos los usuarios de la wiki en tiempo real.

En relación con los conocimientos adquiridos, el 100\% de los encuestados afirma saber diferenciar un blog de una wiki. Destacan del blog la aparición de la información más actual en su orden cronológico, la autoría personal publicando entradas que sólo puede modificar el administrador (frente a la colectividad de la wiki), su falta de dinamismo y de interacción característicos de la wiki. Recuerdan que los blogs facilitan información útil según el criterio de los creadores para solventar dudas que ellos mismos pudieron tener en el pasado. Mientras que las wikis "reversionan" esas dudas cambiando la entrada en tiempo real.

Otros aspectos a destacar fueron: el trabajo colaborativo, el acceso en línea y con actualización en tiempo real, la capacidad para el intercambio y creación colectivo de conocimiento revisando, editando y adjuntando nuevas informaciones (definiciones, enlaces a otras páginas web, bibliografías especializadas, etc.), la potencialidad para ayudar en el trabajo del resto de miembros del grupo. 
Se llegó a una puntuación de 4,3 sobre 5 en la valoración relativa a la ayuda de las wikis en la traducción y revisión colectivas; y también en los contenidos incluidos en la wiki y en el listado de blogs especializados en traducción ayudará al alumno en su futura profesión.

En general los estudiantes se mostraron satisfechos con los contenidos de la wiki que calificaron de lógicos y relevantes; y consideraron como suficiente actualizarlo periódicamente. A excepción de una persona que declaró difícil la forma de introducir los datos y de otra que percibía excesivo el número de blogs incluidos en la wiki.

A nivel técnico sí exigen poder cambiar el diseño de la wiki e incluso que permanezca visible el nombre de la persona que editó la entrada (en nuestra profesión el nombre de la persona que tradujo o revisó). Fue tal el compromiso adquirido que sugirieron la posibilidad de incluir más contenidos en torno a la profesión como:

1. Información teórica sobre diferentes métodos de traducción p.e. Kussmaul

2. Más idiomas de destino en las traducciones

3. Más tipos de traducciones

4. Compartir recursos de otras especialidades (ej. Jurídica)

5. Un glosario específico para cada proyecto de traducción y dificultades de su traducción

Hay que destacar la mejora en la competencia evaluativa solicitando crear algún sistema de control que impida subir a la red información errónea y tomando conciencia de la necesidad de contrastar las fuentes de información e incluir referencias bibliográficas en cada entrada de la wiki reconociendo la autoría.

Los encuestados manifiestan unánimemente un alto grado de satisfacción con esta experiencia y donde la metodología de trabajo en la wiki podría aplicarse a otras materias de la carrera. Valoran esta herramienta como de gran ayuda para cualquier traducción colectiva y tener un espacio para compartir información y recursos de manera sencilla y accesible para todos/as. Incluso extienden el uso de la wiki al área de interpretación contrastando expresiones cooperativamente, incluyendo aclaraciones, vocabulario especializado, diccionarios consultados... En definitiva, el trabajo del traductor y del intérprete también requiere de un trabajo en equipo donde la wiki resulta ser una herramienta altamente valorada tras este experimento.

Este interés también se vio reflejado en la calificación final de la asignatura ya que el $100 \%$ de los participantes superó con una media de notable la asignatura en la primera convocatoria.

\section{Conclusiones}

Esta experiencia ha sido altamente positiva mejorando las habilidades comunicativas e interpersonales de los estudiantes, y potenciando el teletrabajo colaborativo, autoaprendizaje y la interdisciplinariedad. Para algunos ha sido la primera toma de contacto con herramientas cooperativas Web 2.0 y les ha hecho tomar conciencia de su importancia. Descubren que la realidad laboral exige un conocimiento del método de traducción y también de Web 2.0 para ser competentes y desempeñar su futura profesión de la manera más adecuada facilitándoles su integración en el mercado de trabajo.

Con TradWiki se ha logrado una mayor comprensión de lo que es una wiki a través de su uso. Es evidente que la heterogeneidad de inquietudes forzara a establecer el carácter obligatorio de algunas actividades. Pero la inclusión de apartados algo más lúdicos, como humor y vídeos, redujo el rechazo de la wiki por parte de los más reacios y motivó al resto a una mayor actividad y aportación de información. Además este éxito se debe también al interés de este grupo de personas en participar en métodos de aprendizaje más experimentales basados en el trabajo continuo y cooperativo.

Hay que resaltar que TradWiki impulsó la ampliación de conocimientos buscando información sobre servicios de la Web 2.0, como tutoriales, identificación de otros programas 
y/o plataformas, etc. Paralelamente, permitió que los participantes aprendieran, de una forma aplicada y dinámica a:

1. Seleccionar información relevante a partir del análisis y valoración del contenido de blogs especializados.

2. Generar conocimientos colectivos y compartir nuevas informaciones en forma de humor o de terminología a través de la wiki principalmente, o también del foro.

3. Potenciar la iniciativa y curiosidad personal. Algunos miembros de la clase llegaron a plantear la creación de otra wiki para generar un estudio colectivo de la asignatura poniendo en común los apuntes.

Pese a la sencillez en la utilización de la plataforma, algunos estudiantes tuvieron dificultades en la edición de wikis. Esta situación se debió al desconocimiento de la herramienta. También consideraron un inconveniente la imposibilidad de editar simultáneamente una entrada. No obstante resulta del todo justificable ya que, en caso contrario, no se podría controlar la versión más actual.

Se refuerza la interiorización de principios y valores poco cuantificables pero verdaderamente importantes en el trabajo del traductor. Son conscientes de que cualquier trabajo colectivo requiere más compromiso, implicación y responsabilidad ya que se pone en riesgo la labor de otros colaboradores. Si se es miembro de un grupo, el trabajo de los compañeros depende de la seriedad y honestidad con se haya realizado el propio. Este rigor se traslada a la hora de contrastar las fuentes y de identificar al autor/a de cada entrada para valorar el grado de fiabilidad de la información.

Como conclusión, se comprueba el sentido práctico de este proyecto tanto cuantitativamente como a través de las apreciaciones del alumnado. Y se confirma que los recursos Web 2.0 permiten aumentar el abanico de posibilidades a la hora de educar en el trabajo telemático y cooperativo con vistas a una formación de profesionales capacitados. Esta iniciativa puede servir de modelo a otras experiencias y a la mejora de la formación en la Universidad.

\section{Bibliografia}

Alcina Caudet, A. (2002) "Estrategias y recursos en la enseñanza de la informática aplicada a la traducción". Papers de Tradumática: Actes del Primer Simposi sobre l'Ensenyament a distància i semipresencial de la Tradumàtica. Bellaterra, 6 i 7 de juny. Disponible en línea:<http://ddd.uab.cat/pub/poncom/2002/64984/estrecensinftraiSPA.pdf>. Fecha de consulta de la página: 06.06.2013

Bold, M. (2006). "Use of wikis in graduate course work". Journal of Interactive Learning Research, 17(1), 5-14. Disponible en http://search.proquest.com/docview/211275081? accountid=14542 Fecha de consulta de la página: 02.06.2016).

Churches, A. (2009). Bloom's Digital Taxonomy. Disponible en línea : $<$ http://http://edorigami.wikispaces.com/file/view/bloom\%27s\%20Digital\%20taxo nomy \%20v3.01.pdf/65720266/bloom\%27s\%20Digital\%20taxonomy\%20v3.01.p df>. Fecha de consulta de la página: 10.07 .2013 )

Cunningham, Ward. (1995). "History of the Wiki". [en línea] Recuperado de http://c2.com/cgi/wiki?WikiHistory Fecha de consulta de la página: 01.06.2016.

Duffy, Peter and Bruns, Axel (2006) "The Use of Blogs, Wikis and RSS in Education: A Conversation of Possibilities". En Proceedings Online Learning and Teaching Conference 2006, pages pp. 31-38, Brisbane. Disponible en línea: http://eprints.qut.edu.au. Fecha de consulta de la página: 25.05.16.

Franklin, T. Y Van Harmelen, M. (2007) Web 2.0 for content for Learning and Teaching in Higher Education. Bristol: JISC. Disponible en línea:

<http://www.jisc.ac.uk/media/documents/programmes/digitalrepositories/web2-contentlearning-and-teaching.pdf>. Fecha de consulta de la página: 31.07.13. 
General Secretariat of the Council (2012). The Language Service of the General Secretariat of the Council of the European Union: Making Multilingualism Work. Bruselas: General Secretariat of the Council. Disponible en línea:

<http://www.consilium.europa.eu/media/1566791/qc-32-11-696_en_web.pdf> doi:10.2860/79193. Fecha de consulta de la página: 3-7-2013)

Kussmaul, P. 1995. Training the Translator. Amsterdam: John Benjamins.

Lavin, R. y Claro, J. (2005) 'Wikis as constructivist learning environments'. JALT CALL 2005 Proceedings p. 9-13.

Leuf, B y Cunningham, W. (2002) "What Is Wiki" [en línea] Recuperado de http://www.wiki.org/wiki.cgi?WhatlsWiki Fecha de consulta de la página: 01.06.2016.

Martín, E. y Soler, J. (2005). "MLV: Multilingual Vendors". Revista de Tradumática, 4, (decembre). Disponible en línea: <http://www.fti.uab.es/tradumatica/revista/num3/articles/05/05.pdf>. Fecha de consulta de la página: 20.06.13.

Moodle. Página oficial de Moodle [en línea] https://moodle.org/ Fecha de consulta de la página: 20.05.16.

Olvera Lobo, M.D. et al (2004). "Aplicación de un modelo didáctico de enseñanza de la traducción”. Eslavística complutense, 4 p. 249-255.

Olvera Lobo, M.D. et al (2007). "A profesional approach to translator training (PATT)". Meta: Journal des traducteurs, 52 (3) p. 517-528.

Penney, S. (2012). Bloom's taxonomy. Disponible en línea:

$<$ http://faculty.indstate.edu/spenney/bdt.htm>. Fecha de consulta de la página: 10.07.13.

Pérez González, L. y Rico Pérez, C. (2001). "Enhancing process-research in translation training: Towards a collaborative management of classroom dynamics", en: Cruz, I.; Santamaría, C. et al (eds.) La lingüística aplicada a finales del siglo XX, ensayos y propuestas. Ensayos y propuestas. vol. II. Alcalá de Henares: Servicio de publicaciones de Alcalá, 861-864.

Polo, L. y Ferrer Mora, H. (2010) "Aplicación de las TIC en traducción e interpretación en la Universidad de Valencia: experiencias y reflexiones". Redit, 4:23-41. Disponible en línea : <http://www.redit.uma.es/Archiv/n4/mono_LauraRamirezPolo_redit4.pdf>. Fecha de consulta de la página: 30.06 .13

Sigala, M. (2007). "Integrating Web 2.0 in e-learning environments: a socio-technical approach". International Journal of Knowledge and Learning, 3(6): 628-648. Citado por: Romero Frías, E. (2010) "El empleo de wikis en la docencia universitaria: resultados de una experiencia en Contabilidad".

Educade: Revista de Educación en Contabilidad, Finanzas y Administración de Empresas. The Spanish Journal of Accounting, Finance and Management Education, 1(1) p. 4358 Disponible en línea: <http://http://www.educade.es/docs/01/04-romero.pdf>. Fecha de consulta de la página: 02.06.13.

Sturm, M., KennelL, T., Mcbride, R. y Kelly, M. (2009). "The Pedagogical Implications of Web 2.0", en Thomas, M. (ed.) Handbook of Research on Web 2.0 and Second Language Learning. Hershey, PA: Information Science Reference. P. 367-384.

Zayas, F. (2006) "Mis primeras Experiencias con los Blog en el Aula". En Quaderns Digitals: Revista de Nuevas Tecnologías y Sociedad, ISSN 1575-9393, №. 42 